\title{
Implications for Discovery of Strong Radial Magnetic Field at the Galactic Center-Challenge to Black Hole Models
}

\author{
Qiuhe Peng ${ }^{1}$, Zheng Li ${ }^{2}$ \\ ${ }^{1}$ School of Astronomy and Space Science, Nanjing University, Nanjing, China \\ ${ }^{2}$ Xinjiang Astronomical Observatory, University of Chinese Academy of Sciences, Urumqi, China \\ Email: qhpeng@nju.edu.cn, lizheng@xao.ac.cn
}

How to cite this paper: Peng, Q.H. and Li, Z. (2019) Implications for Discovery of Strong Radial Magnetic Field at the Galactic Center-Challenge to Black Hole Models. Journal of Modern Physics, 10, 1416-1423. https://doi.org/10.4236/jmp.2019.1012094

Received: September 10, 2019

Accepted: October 26, 2019

Published: October 29, 2019

Copyright () 2019 by author(s) and Scientific Research Publishing Inc. This work is licensed under the Creative Commons Attribution International License (CC BY 4.0).

http://creativecommons.org/licenses/by/4.0/ (c) (i)

Open Access

\begin{abstract}
The black hole model will be excluded by a very strong radial magnetic field near the Galactic Center which has been detected in 2013. Following it, the explosion mechanism, for both supernova and the hot big bang of the Universe, driven by magnetic monopoles is proposed in this paper.
\end{abstract}

\section{Keywords}

Black Hole Model, Strong Radial Magnetic Field, Magnetic Monopoles

\section{Two Dilemmas}

The radial magnetic field near the Galactic Center (GC) has been detected in 2013 [1].

$$
B \geq 8\left(\frac{n_{e}}{26 \mathrm{~cm}^{-3}}\right)^{-1} \mathrm{mG}(\text { at } r \approx 0.12 \mathrm{pc}) .
$$

where $n_{e}$ is the number density of electrons. The first question is what is the role of the strong radial magnetic field? It is well known that a charged particle is hard to transfer cross the magnetic field line by the Lorentz force. According to the principle of the Alphen magneto-hydrodynamic freezing effect, the accretion (plasma) disk will be prevented from approaching to the GC by the strong radial magnetic field when the magnetic field is stronger more than the Alphen critical magnetic field

$$
B>B_{\text {Alphen }}=1.3\left(\frac{n_{e}}{26 \mathrm{~cm}^{-3}}\right)^{1 / 2} \mathrm{mG} \quad(\text { at } r \approx 0.12 \mathrm{pc})
$$


(The mass of the central supermassive object is taken as $4.6 \times 10^{6} M_{\odot}$ ).

And the accretion (plasma) disk may not enter in the neighborhood of the GC. Thus the radiations observed [2] from the GC direction are hardly emitted by the gas of accretion disk around the black hole $(\mathrm{BH})$ of the GC which is the popular idea. This is a dilemma of the standard accretion disk model of $\mathrm{BH}$ in the GC [2] [3] [4].

The second question is what is the origin of the strong magnetic field near the $\mathrm{GC}$ ?

It is well known that the most efficiency dynamo of producing magnetic field up to now is the advanced $\alpha$-turbulence dynamo in the sunspot (proposed by Parker) [5].

But the resulting magnetic field produced by the similar $\alpha$-turbulence dynamo in the interstellar gas is only $B \leq 0.1 \mu \mathrm{G}$ at $r \approx 0.12 \mathrm{pc}$, which is five-order of magnitude less than the observed lower limit for the field strength, $8 \mathrm{mG}$. The observed magnetic field with a lower limit of $8 \mathrm{mG}$ near the GC is hardly produced by so far known generator mechanism. This is the second dilemma.

To sum up, we conclude that the discovery of abnormally strong radial magnetic fields at the center of the GC presents the two dilemmas above for astrophysics.

However, these two dilemmas may simultaneously be solved by our model "Super-massive star with magnetic monopoles" [6] for quasars and active galactic nuclei (AGNs) (including the super-massive object at the GC) proposed since 1985. The discovered strong radial magnetic field near the GC is just consistent with our prediction on quantity.

\section{A Supermassive Star Model with Magnetic Monopoles (SMSMM)}

Taking the RC effect, that the MM may catalyze a nucleon decay, as an energy source, however, this dilemma in the GC may be naturally solved by our SMSMM at the GC (non BH). Three predictions, at least, in our model [6] are quantitatively confirmed by astronomical observations afterwards: 1) The radial magnetic field produced by the MMs condensed in the core of the super-massive object is about $B \approx(10 \sim 50) \mathrm{mG}$ at $r=0.12 \mathrm{pc}$ which is consistent with the lower limit of the observed magnetic field. This is a key prediction. All other models will be excluded when the key prediction is confirmed by the astronomical observation.

2) Plenty of positrons are emitted from the GC, the production rate is $10^{43}$ $\mathrm{e}^{+} / \mathrm{sec}$ or so. This prediction is consistent with high-energy astrophysical observations [7] in 2003.

3) We predicted the surface temperature of the super-massive stellar object at the galactic center to be $123 \mathrm{~K}$ and the corresponding $10^{13} \mathrm{~Hz}$ (at the sub-millimeter range), and this is quite close to the observed value of $10^{12} \mathrm{~Hz}$ [8].

These observations may show the signals for existence of MMs such as the supermassive object at the GC. 
To sum up, we conclude three important significances of our predictions above have been confirmed by the astronomical observations:

1) The Black Hole model in the Galactic Center is nonphysical.

2) These are the astrophysical evidences for the existence of MMs.

I hereby declare that astronomical observations are actually physical experiments in space.

3) Our model is reasonable. Especially the RC effect may be real and we may use it as an energy source for the supernova explosion and for the Earth core.

\section{Query on the BH Models for Quasars and AGNs}

It is now generally believed by most astronomers that bright quasars observed at large redshift (for example, $z>1$ or even $z>6$ ) are supermassive black holes formed in the primordial universe.

The spectacularly huge luminosity is supplied by the matter of a rotating accretion disk around the $\mathrm{BH}$. We point out that the most serious contradiction in the $\mathrm{BH}$ model comes from the mass of the quasars original black hole.

We asked to compare birth in the early universe. In order to find the original mass of a quasar when it was born in the early universe, we need to subtract the mass added by accretion from the mass of the quasar determined today from the time of its birth $(t \approx 0)$ to the time of $t(z)$. However, according to various possible accretion theories in the current research, taking the data of 105,783 quasars from [9] which are based by SLOAN Digital sky survey (SDSS), we find that the mass of these black holes (quasars) with medium and low redshift is mostly negative or very small after deduction (see Figure 1). This is totally ridiculous.

However, the situation is totally different when we take our model (SMSMM). The mass of the supermassive object must decrease gradually due to the baryons decaying catalyzed by the MMs. And the nucleon decaying products (including the pions, muons, positrons and the radiation) would go out of the object continuously.

In order to compare the mass of the quasars (active galactic nuclei) observed at different redshift in the primordial era, the mass lost during their evolution due to radiation by the nucleon decay catalyzed by MMs. should be added into the mass of the quasars (AGNs) observed at different redshift now.

Adding the lost mass during their evolution, the statistic distribution of number of the quasars (AGNs) with their primordial mass of shows a Gauss distribution (see Figure 2). It is reasonable.

\section{Supernova Explosion Driven by MMs}

What role played by the MM in supernova (SN) explosion?

The MMs in the interior of stars and planets are almost by captured from space during their life time after their formation. The total number of MMs captured from space by the progenitors of SN after their formation may be estimated to be 


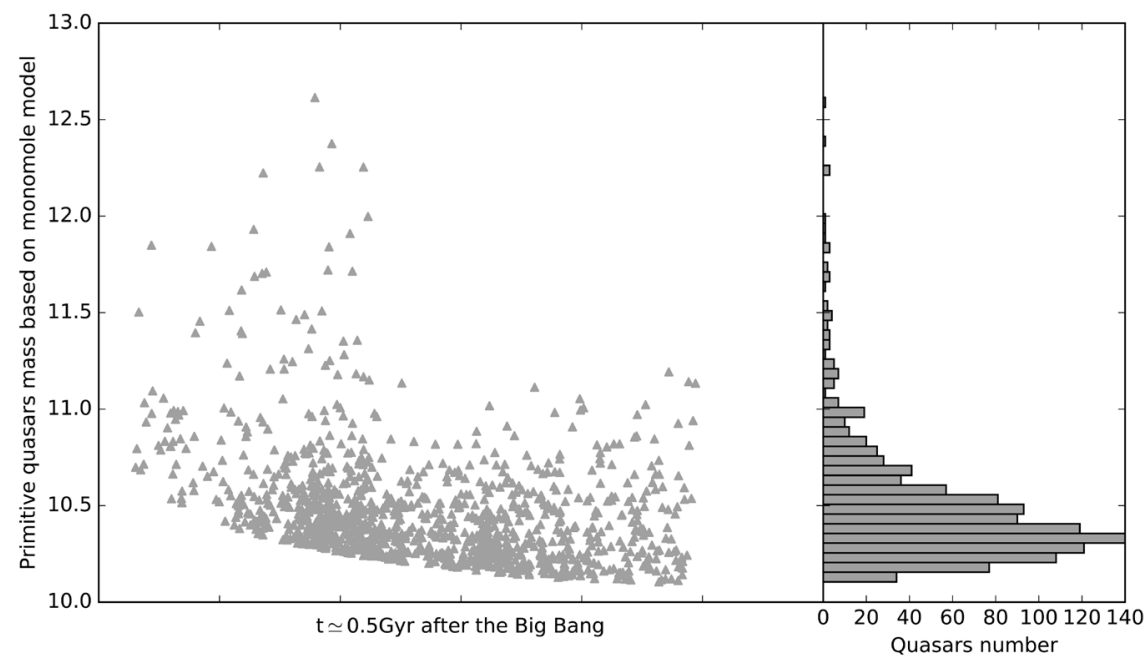

Figure 1. Distribution of the mass of primordial mass of quasars in black hole model, the solid line represents the quasar mass error not considered in the statistics, dash-dot line and dotted line represent the upper and lower limits of the quasar mass error are considered separately in the statistics (Data for 105,783 quasars are from [9]), the result is from Zheng Li and Ming Zhang's work (in preparing).

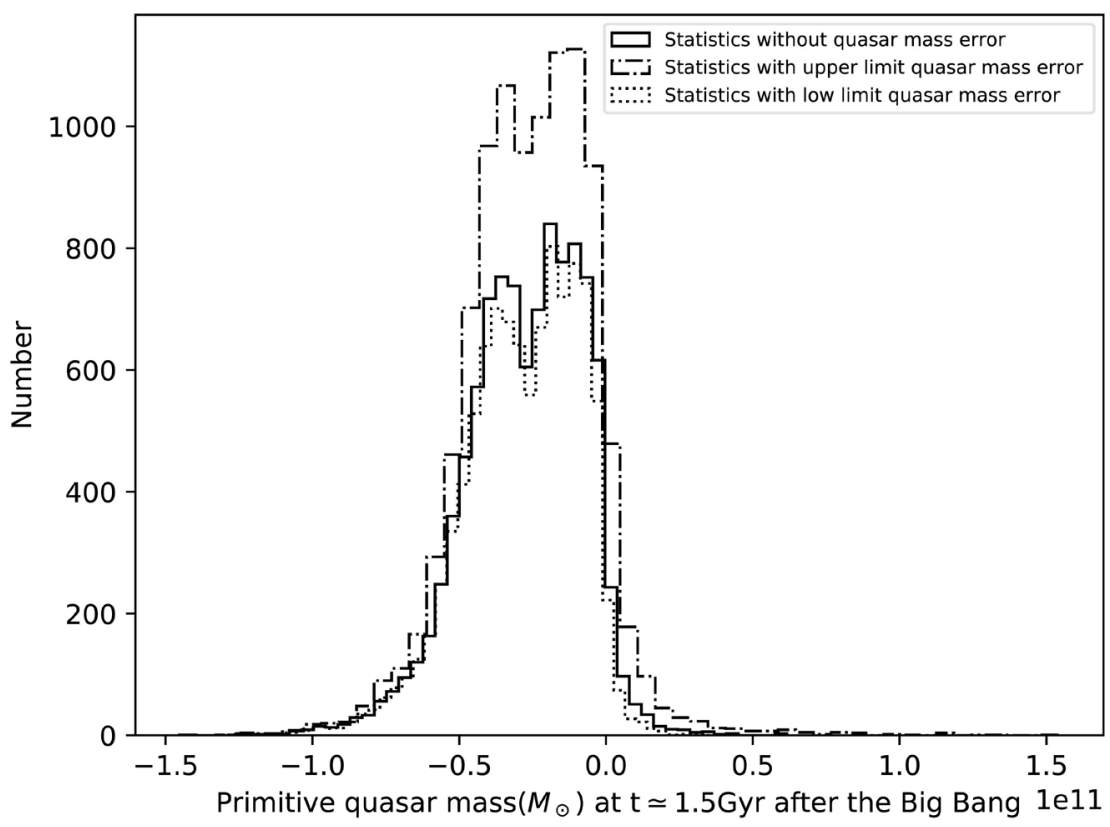

Figure 2. Distribution of the primordial mass of quasars in the model of supermassive star with magnetic monopoles, the quasar masses we take log10 (Data for 105,783 quasars are from [9]), the result is from Zheng Li and Ming Zhang's work (in preparing).

$$
N_{m}=4 \pi R^{2} \Phi_{m} t \approx 1.0 \times 10^{31}\left(\frac{\Phi_{m}^{(0)}}{10^{-2} \Phi_{m}^{(u p)}}\right)\left(\frac{R_{R G}}{10^{3} R_{\odot}}\right)^{2}\left(\frac{t_{R G}}{10^{6} \mathrm{Yr}}\right)
$$

where $R$ denotes the radius of the star (or planet) and $t$ is the life time of the star (or planet), $\Phi_{m}$ is the flux of the MM flight in the space. The superscript (0) is the sign of its value in the space. The number of MMs captured from space by 
the progenitor of SN is mainly obtained in its red giant stage due to the radius of corresponding red giant being hundreds of times larger than the radius of the corresponding main sequence star, although the life time of its red giant stage is about $1 / 10$ of times shorter than the life time of its corresponding main sequence star. $\Phi_{m}^{(u p)}$ is the up limit of the flux for the MM flight in the space (Parker, 1970) [10].

$$
\Phi_{m}^{(u p)} \approx\left(10^{-12} \sim 10^{-16}\right) \mathrm{cm}^{-2} \cdot \mathrm{s}^{-1} \cdot \mathrm{sr}^{-1}
$$

Taking the RC effect as the energy source, the luminosity of the supernova is.

$$
\begin{aligned}
& L_{m} \approx 2.5 \times 10^{43} a\left(\frac{\xi}{100}\right)\left(\frac{n_{B}^{(c)}}{n_{n u c}}\right)\left(\frac{T_{c}}{10^{11} \mathrm{~K}}\right)^{1 / 2} \mathrm{erg} / \mathrm{s} . \\
& a=\left(\frac{R_{R G}}{10^{3} R_{\odot}}\right)^{2}\left(\frac{t_{R G}}{1 \times 10^{6} \mathrm{Yr}}\right) . \\
& \xi \equiv\left(\frac{\sigma}{10^{-30} \mathrm{~cm}^{2}}\right)\left(\frac{\Phi_{m}^{(0)}}{10^{-2} \Phi_{m}^{(u p)}}\right) .
\end{aligned}
$$

where $n_{B}^{(c)}$ is the number density of the baryons in the center for the core of the $\mathrm{SN}, n_{n u c}$ is the nuclear (number) density, $\sigma$ is the cross section of the RC effect. For collapsed supernova, its central density increase with the core mass of the collapsing supernova we use some approximate (reasonable) estimates:

$$
\begin{gathered}
t_{R G}=10^{6}\left(\frac{M}{20 M_{\odot}}\right)^{-1} \mathrm{Yr} \\
\frac{R_{R G}}{R_{\odot}}=10^{3}\left(\frac{M}{20 M_{\odot}}\right)^{\beta}, \quad \beta \approx(1.0 \sim 1.5) .
\end{gathered}
$$

Hence

$$
\begin{gathered}
a \approx\left(\frac{M}{20 M_{\odot}}\right)^{(2 \beta-1)} . \\
n_{B}^{(c)} / n_{n u c} \propto\left(M_{c} / 20 M_{\odot}\right)^{2} .
\end{gathered}
$$

The relationship between the peak luminosity of a supernova and the mass of its progenitor may be written as

$$
L_{m}^{(\text {peak })} \approx 5.0 \times 10^{43}\left(\frac{M}{20 M_{\odot}}\right)^{2 \beta+1}\left(\frac{\xi}{100}\right)\left(\frac{T_{c}}{10^{11} \mathrm{~K}}\right)^{1 / 2} \mathrm{ergs} / \mathrm{s} .
$$

The supernova will explode when the peak luminosity of the supernova is higher than the Eddingtons luminosity which is the critical luminosity of a stable star:

$$
\begin{gathered}
L_{\text {peak }}>L_{E d d} . \\
L_{E d d}=\frac{4 \pi c G M}{\kappa} \approx 1.3 \times 10^{38}\left(\frac{\kappa}{0.4}\right)^{-1}\left(\frac{M}{M_{\text {sun }}}\right) \mathrm{ergs} / \mathrm{s} .
\end{gathered}
$$


We can use the ratio, $b$ to measure the magnitude of the supernova explosion.

$$
\begin{gathered}
b \equiv L_{\text {peak }} / L_{E d d} . \\
b \approx 2 \times 10^{4}\left(\frac{M}{20 M_{\odot}}\right)^{2 \beta} .
\end{gathered}
$$

For the super luminosity supernova ASASSN-15lh (Sep. 12015), $L=2.2 \times 10^{49} \mathrm{ergs} / \mathrm{s}$ at the $15^{\text {th }}$ day after the peak, it is easily explained by an assumption: 1) Its progenitor with the initial mass more than $10^{4} M_{\odot}$, and its radius $R \approx 10^{4-5} R_{\odot}, t \approx 10^{4} \mathrm{Yr} .2$ ) The number density of the baryons in the center for the core of the SN during its collapse is reach to $n_{B}^{(c)} \approx 10^{3} n_{n u c}$.

The SN may be very weak without strong explosion when its RC luminosity is not much higher than the Eddington's luminosity, $L_{E d d} \approx 1.3 \times 10^{38}\left(M / M_{\odot}\right)$.

(Such as Cas A and the G1.9 + 0.3). The Equation (12) is the relationship of the peak luminosity of $\mathrm{SN}$ with the mass of their progenitors.

Most of the MMs will be rapid thrown outwards with the plasma by the strong Coulomb electromagnetic interaction, and they will go away from the stars. However, trace MMs will return back to the core of the star. The residual MM still continue to catalyze the nucleons decaying. The corresponding radiation pressure is outward against material collapse.

When the RC luminosity catalyzed by the MMs in the core is less than the Eddington's luminosity of the remnant, they will reach at the dynamic equilibrium. Neither material is pushed outward by the radiation pressure, nor will the core collapse to the center. The central density cannot tend to infinite and the SN remnant is not a black hole. It means that no black holes with stellar mass formed through supernova explosion of massive stars.

Using the same idea we may also explain naturally following two mysteries: 1) why the Earth's core is in a melting state. The parameter value in the Equation (5) and (12), Equation $\xi \approx 100$, is estimated based on the measured outward heat flow from the earth's core and on the energy yield rate of nucleon decay catalyzed by magnetic monopoles (RC effect); 2) why no white dwarf with surface temperature lower than $10^{3} \mathrm{~K}$ has not been observed up to now?

\section{Physics on the Hot Big Bang of the Universe Driven by MMs}

On the standard model of the hot big bang cosmology, the early universe is depicted by extrapolating back to a hot and dense initial state of Planck length and Planck time derived with the help of the uncertainty principle. However, the formation of the big bang itself has not been investigated, i.e. nobody gives answer to the question what is the physical reason of Hot Big Bang of the Universe?

Using the same idea that nucleons may decay catalyzed by the MM with strong interaction cross section (i.e. RC effect), we may also explain naturally the physical reason of Hot Big Bang of the Universe.

It is generally estimated and believed that there are $2.0 \times 10^{11}$ galaxies. Every 
galaxy is roughly the size of over Milky galaxy with $10^{11}$ stars, then the total number of stars in the Universe is about $10^{23}$. The mass of the sun is $2.0 \times 10^{33}$ gram, then the total mass of the Universe of the baryons is $2.0 \times 10^{56}$ gram and the total number of the baryons is $1.0 \times 10^{80}$. If the content of the magnetic monopoles of the same polarity contained in the Universe is $\xi=N_{m} / N_{B}=10^{-20}\left(\xi / \xi^{(u p)}\right)$, here $N_{m}$ and $N_{B}$ are the number of magnetic monopole and baryons, respectively. $\xi^{(u p)}$ is the Parker up limit (Parker, 1970), and $\xi^{(u p)} \approx 10 \sim 20$. So the total number of the magnetic monopoles of the same polarity contained in the universe may be estimated to be $N_{m}=10^{60}\left(\xi / \xi^{(u p)}\right)$ (Peng and Chou, 2001; Peng et al., 2017). The magnetic monopoles in the high temperature baryon plasma are strongly compressed and moving very fast toward the center via electromagnetic interaction. The RC luminosity produced by nucleon decay which is catalyzed by the magnetic monopoles is given by

$$
L_{m}=N_{m} n_{B}^{(c)}\left\langle\sigma v_{T}\right\rangle m_{B} c^{2} \approx 10^{75}\left(\frac{n_{B}}{n_{\text {nuс }}}\right)\left(\frac{\xi}{\xi^{(u p)}}\right)\left(\frac{\sigma_{(R C)}}{10^{-80} \mathrm{~cm}^{2}}\right) \mathrm{ergs} / \mathrm{sec} .
$$

when the total mass in the universe is compressed to become super-massive body, the corresponding Eddington luminosity is given by

$$
L_{E d d}=10^{38}\left(\frac{M}{M_{\odot}}\right) \mathrm{ergs} / \mathrm{sec} \approx 10^{61} \mathrm{ergs} / \mathrm{sec} .
$$

If the whole universe is compressed such that

$$
\left(\frac{n_{B}^{(c)}}{n_{\text {пис }}}\right)>10^{-10}\left[\left(\frac{\xi}{\xi^{(u p)}}\right)\left(\frac{\sigma_{R C}}{10^{-80} \mathrm{~cm}^{2}}\right)\right]^{-1} .
$$

then $L_{m}>10^{4} L_{E d d}$ and the whole Universe must violently explode outward leading naturally to the hot big bang. This is just the physical mechanism for Hot Big Bang of the Universe.

In view of this, we may propose an oscillating model of the Universe between the expansion phase of the big bang and then the contracting phase by the gravitational attraction.

\section{Conclusion}

In the traditional standard hot big bang cosmology, it is extrapolated back to the initial singularity of the universe. This is done purely by theoretical speculation. Our model of the hot big bang is obtained in terms of the Rubakov-Callan luminosity and no other theoretical arguments or anticipation is required. In our model, the expression phase may finally be ended and followed by the contraction phase due to gravitational attraction.

\section{Acknowledgements}

This work was supported by the National Basic Research Programme of China (2015CB857100), the National Science Foundation of China (11773062) and the West Light Foundation of Chinese Academy of Sciences (2017-XBQNXZ-A-008). 


\section{Conflicts of Interest}

The authors declare no conflicts of interest regarding the publication of this paper.

\section{References}

[1] Eatough, R.P., Falcke, H., Karuppusamy, R., Lee, K.J., Champion, D.J., Keane, E.F., Desvignes, G., Schnitzeler, D.H.F.M., Spitler, L.G., Kramer, M., et al. (2013) Nature, 501, 391. https://doi.org/10.1038/nature12499

[2] Peng, Q.-H., Liu, J.-J. and Chou, C.-K. (2016) Astrophysics and Space Science, 361, 388. https://doi.org/10.1007/s10509-016-2977-8

[3] Peng, Q.-H., Liu, J.-J. and Ma, Z.-Q. (2017) New Astronomy, 57, 59-62. https://doi.org/10.1016/j.newast.2017.06.011

[4] Peng, Q.-H., Liu, J.-J. and Chou, C.-K. (2017) Astrophysics and Space Science, 362, 222. https://doi.org/10.1007/s10509-017-3201-1

[5] Charbonneau, P. (2010) Living Reviews in Solar Physics, 7, 3. https://doi.org/10.12942/lrsp-2010-3

[6] Peng, Q.-H. and Chou, C.-K. (2001) The Astrophysical Journal Letters, 551, L23. https://doi.org/10.1086/319824

[7] Lebrun, F., Terrier, R., Bazzano, A., Bélanger, G., Bird, A., Bouchet, L., Dean, A., Del Santo, M., Goldwurm, A., Lund, N., et al. (2004) Nature, 428, 293. https://doi.org/10.1038/nature02407

[8] Falcke, H. and Markoff, S.B. (2013) Classical and Quantum Gravity, 30, Article ID: 244003. https://doi.org/10.1088/0264-9381/30/24/244003

[9] Shen, Y., Richards, G.T., Strauss, M.A., Hall, P.B., Schneider, D.P., Snedden, S., Bizyaev, D., Brewington, H., Malanushenko, V., Malanushenko, E., et al. (2011) The Astrophysical Journal Supplement Series, 194, 45. https://doi.org/10.1088/0067-0049/194/2/45

[10] Parker, E.N. (1970) The Astrophysical Journal, 160, 383. https://doi.org/10.1086/150442 